\title{
Therapeutic Characteristics, Chemotherapy-Related Toxicities and Survivorship in Colorectal Cancer Patients
}

\author{
Zineb Aoullay ${ }^{1 *}$, Meriem Slaoui ${ }^{2}$, Rachid Razine ${ }^{3,4}$, Abdelouahed Er-Raki ${ }^{5}$, \\ Bouchra Meddah $^{1}$, Yahia Cherrah ${ }^{1}$
}

\footnotetext{
OPEN ACCESS

Citation: Zineb Aoullay, Meriem Slaoui, Rachid Razine, Abdelouahed Er- Raki, Bouchra Meddah, Yahia Cherrah. Therapeutic Characteristics, Chemotherapy-Related Toxicities and Survivorship in Colorectal Cancer Patients. Ethiop J Health Sci. 2020;30(1):65.doi: http:// dx.doi.org/ 10.4314/ejhs.v30i1.9

Received: September 13, 2019

Accepted: October 07, 2019

Published: January 1, 2020

Published: January 1, 2020

Copyright: (C) 2019 Zineb A., et al. This is an open access article distributed under the terms of the Creative Commons Attribution License, which permits unrestricted use, distribution, and reproduction in any medium, provided the original author and source are credited. Funding: Nil

Competing Interests: The authors declare that this manuscript was approved by all authors in its form and that no competing interest exists.

Affiliation and Correspondence:

${ }^{1}$ Laboratory of Pharmacology and

Toxicology, Faculty of Medicine and

Pharmacy of Rabat, University

Mohamed V Rabat, Avenue

Mohammed Belarbi El Alaoui - Souissi

- BP, 6203 Rabat, Morocco

${ }^{2}$ Faculty of Medicine and Pharmacy of Rabat, University Mohamed V Rabat, Avenue Mohammed Belarbi El Alaoui Souissi - BP, 6203 Rabat, Morocco

${ }^{3}$ Laboratory of Biostatistics,

Epidemiology and Clinical Research, Université Mohamed V-Souissi Faculty of Medicine and Pharmacy of Rabat, Avenue Mohammed Belarbi El AlaouiSouissi, BP 6203 Rabat, Morocco

${ }^{4}$ Department of Public Health, Université Mohamed V-Souissi Faculty of Medicine and Pharmacy of Rabat, Avenue Mohammed Belarbi El Alaoui Souissi, BP 6203 Rabat, Morocco

National Institute of Oncology, Rabat, Morocco

*Email: zinebaoullay@gmail.com
}

\author{
ABSTRACT
}

BACKGROUND: Colorectal Cancer (CRC) is a major health problem around the globe. In Morocco, the disease ranks third after breast and lung cancers. This study is the first in Morocco to investigate epidemiological, clinical and therapeutic features while exhaustively describing toxic side-effects to chemotherapy of CRC and studying the 3-years survivorship.

METHODS: This is a descriptive and analytical retrospective study of about 290 patients with CRC enrolled during the period of January-December 2013. Statistical analysis was performed to correlate clinicopathological data with chemotherapy toxicity and survivorship in patients, by $\mathrm{Chi}^{2}$ test. Overall Survival (OS) rate has been calculated by the Kaplan-Meier method and compared using Log-rank test.

RESULTS: Fifty-five percent had a tumor localized in rectum, and $42,8 \%$ in colon. Mean age of these patients at diagnosis was 56,16 \pm 14,6. incidence rate of adverse events (grade I to IV) was $85,6 \%$. Diarrhea was the predominant toxicity (4.6\%) occurring at a high grade (grade III-IV).

The 3-years OS rate of patients with CRC was 71\%. OS decreased by age, and patients with age subgroup between 40 to 59 years had a better $O S$ than the other age subgroups (60 to 79 years and $>80$ years) with a p-value of 0.0001 . Occurence of toxicity (all grades and types) was linked to a higher survival rates compared to the group who had no toxicity noticed (p-value of 0.001).

CONCLUSION: Our study shows that patients who had a polychemotherapy had a better OS than those who had monotherapy (p-value of 0.002).

KEYWORDS: Colorectal cancer, chemotherapy toxicities, overall survival (OS)

\section{INTRODUCTION}

Due to its frequency and gravity, colorectal cancer is a major health problem around the globe. It is the third most common cancer worldwide after lung and breast cancers, in terms of incidence. It ranks second in terms of mortality with an estimated 1.8 million new cases diagnosed and 881,000 deaths in 2018 according to Global Cancer Statistics 2018 (1). 
A worldwide distribution shows that incidence rates, influenced by the lifestyle of populations, are elevated in parts of Europe (eg, in Hungary, Slovenia, Slovakia, the Netherlands and Norway), Australia/New Zealand, Northern America and Eastern Asia (Japan and the Republic of Korea, Singapore [in females]). It tends to be low or even rare in most regions of Africa and Southern Asia (1). In Morocco, according to the cancer registry of the Greater Casablanca, colorectal cancer is ranking third after breast and lung cancers (2). It represents $6.7 \%$ of cancer cases registered between 2008 and 2012 with a total of 1628 cases. The standardized rates on the world population and the Moroccan population were respectively 9.6 and 7.8 new cases per 100000 individual (2) notifying an increasing incidence these last years. According to the Rabat population-based cancer registry, 4,096 new cases are expected to be diagnosed in 2020 (3).

In Rabat, the incidence of colorectal cancer remains close to the rates in the countries of North Africa and East Asia but is still significantly lower than rates recorded in developed countries (Americas, Europe, etc.) (3).

Colorectal cancer, historically known with a poor prognosis, has over the years been the subject of much research that has changed its treatment. Chemotherapy remains a cornerstone of treatment for this cancer and for many tumor entities, leading to an increased response rate and patient survival. This benefit is offset by several toxicities with potentially unacceptable life-threatening events and, to identical chemotherapy regimen, patients tolerate and respond differently. Several factors are involved, leading to this inter-individual variability such as genetic variability of metabolic enzymes.

Nowadays, the identification of patients at high risk to encounter toxic adverse events, or at risk of treatment failure or either with high chance to respond to chemotherapy, is still controversial and unclear. Nevertheless, the screening of predictive biomarkers could make a considerable progress in differentiating prospectively between patients in terms of response and tolerance against treatment (4).

The lack of awareness about epidemiology of cancers is a major handicap of their treatment. Therefore, it is vital to increase our understanding of the genetic, environmental and social factors that foster these diseases and influence the responses to their treatments, with the aim of applying this knowledge to effective preventive measures and to what cancer epidemiology is dedicated.

In Morocco, more work is dedicated to colorectal cancer to improve medical practice diagnostically as therapeutically. It is in this context that our work fits, and the main purpose is to study the epidemiological, clinical and therapeutic characteristics of colon and rectum cancer, especially the factors associated with chemotherapy-related toxicities and survivorship in Moroccan patients with colorectal cancer.

\section{PATIENTS AND METHODS}

Study design: This is a descriptive and analytical retrospective study of about 290 patients with colorectal cancer, confirmed by a histological examination. Data were collected from the pathological and clinical records of each patient during the period of January-December 2013. To our best knowledge, this study is the first investigation of a Moroccan colorectal cancer population, of epidemiological, clinical, and therapeutic features notifying and exhaustively describing toxic side effects related to chemotherapy of colorectal cancer and studying 3 years survivorship.

Data collection and study variables: A questionnaire was designed to collect information from patients' medical and clinical records available in the registry of the National Institute of Oncology, so a complete and comprehensive database was made. All demographic, clinical, para-clinical, therapeutic characteristics and treatment outcomes data were extracted for colorectal cancer patients.

Treatment and follow-up: The patients received treatment according to the Mayoclinic protocols. Treatment for colorectal cancer is based on the surgery, radiotherapy and chemotherapy; it depends on the discovering stage of the cancer. Thus, it's best treated with surgery when it's discovered at early stage. Before each chemotherapeutic cycle or chemoradiation, each patient was physically examined and any adverse toxic events were evaluated and graded for severity according to National Cancer Institute's Common Terminology Criteria for Adverse Events (NCI-CTCAE) scales. 
Patients were followed up until January 2017. Overall Survival (OS) was calculated from the date of histological diagnosis to the date of death or the last follow-up. All patients who are not reviewed in the last consultation were contacted again by telephone for survivorship assessment. Overall Survival (OS) was defined by the period from the date of histological diagnosis to the date of the last visit or the date of death, regardless of the cause.

Statistical analysis: Statistical analysis was done by SPSS 22.0 software (IBM Corporation, Armonk, NY, USA). Descriptive clinical data were expressed in percentage or median or mean \pm SD. Statistical analysis was performed to correlate clinicopathological data with chemotherapy toxicity and survivorship in patients, by the $\mathrm{Chi}^{2}$ test. Statistical significance was set at the $p$ value $<0.05$. Overall survival rate was calculated by the KaplanMeier method and compared using the Log-rank test. Patients lost to follow-up were considered as a censored event.

Ethics approval and consent to participate: The local Ethical Committee of Biological Research approved the study under the reference number 409/14. No consent was needed because of the retrospective nature of the study. The present publication does not compromise anonymity or confidentiality or breach local data protection laws.

\section{RESULTS}

Demographic, clinical and therapeutic characteristics of patients: In our series, 290 patients with colorectal cancer were included. The baseline patients' demographic, clinical and paraclinical characteristics are listed in Table 1.

The mean age at diagnosis for patients was $56,16 \pm 14,61$ ranging from 24 to 91 . There was a slight male predominance with $50.5 \%$ of men and $49.5 \%$ of women. In our series, $57 \%$ of those cancers interested the rectum and the colon in $42.8 \%$ of cases (Table 1).

According to Table 1, 189 of the patients underwent surgery, so the operability rate was $65.2 \% ; 70$ patients had the neoadjuvant concomitant chemoradiotherapy (24.1\%) and $146(50.4 \%)$ were on chemotherapy. The majority of the
Table 1: Demographic, Clinical and paraclinical, therapeutic characteristics and outcomes of Moroccan patients with colorectal cancer-about 290 cases.

\begin{tabular}{|c|c|}
\hline Characteristics & $\mathrm{N}(\%)$ \\
\hline Age (mean \pm SD) (years) & $56.16 \pm 14.61$ \\
\hline \multicolumn{2}{|l|}{ Sex } \\
\hline Male & $147(50.5)$ \\
\hline Female & $144(49.5)$ \\
\hline \multicolumn{2}{|l|}{ Localization } \\
\hline Colon & $124(42.8)$ \\
\hline Rectum & $166(57.2)$ \\
\hline \multicolumn{2}{|l|}{ Treatment } \\
\hline Surgical treatment & $189(65.2)$ \\
\hline Radiotherapy (rectal cancer) & $6(2.1)$ \\
\hline \multicolumn{2}{|l|}{$\begin{array}{l}\text { Concomitant chemoradiotherapy } \\
\text { (Rectal cancer) }\end{array}$} \\
\hline Neoadjuvante & $70(24.1)$ \\
\hline Exclusive & $7(2.4)$ \\
\hline \multicolumn{2}{|l|}{ Chemotherapy } \\
\hline Adjuvante & $58(20.0)$ \\
\hline Neoadjuvant & $6(2.1)$ \\
\hline Palliative & $82(28.3)$ \\
\hline Not performed & $144(49.7)$ \\
\hline \multicolumn{2}{|l|}{ Line 1} \\
\hline FOLFIRI/ FOFIRI+bevacizumab & $3(2.1)$ \\
\hline XELIRI/XELIRI+bevacizumab & $1(0.7)$ \\
\hline FOLFOX/FOLFOX+bevacizumab & $29(19.8)$ \\
\hline XELOX/XELOX+bevacizumab & $107(73.3)$ \\
\hline XELODA/XELODA+bevacizuma & $6(4.1)$ \\
\hline \multicolumn{2}{|l|}{ Line 2} \\
\hline FOLFIRI/ FOFIRI+bevacizumab & $4(2.8)$ \\
\hline XELIRI/XELIRI+bevacizumab & $34(23.3)$ \\
\hline XELOX/XELOX+bevacizuma & $3(2.1)$ \\
\hline Monchemotherapy & $4(2.7)$ \\
\hline Polychemotherapy & $142(97.3)$ \\
\hline \multirow{2}{*}{\multicolumn{2}{|c|}{ Toxicity (treatment outcomes) }} \\
\hline & \\
\hline Yes & $125(85.6)$ \\
\hline No & $21(14.4)$ \\
\hline \multicolumn{2}{|l|}{$\begin{array}{l}\text { Dose reduction/delay/discontinuation/ } \\
\text { withdrawal } \\
\text { (due to toxicity) }\end{array}$} \\
\hline Drug dose reduction & $9(6.2)$ \\
\hline $\begin{array}{l}\text { Premature discontinuation (ending } \\
\text { treatment) }\end{array}$ & $3(2.1)$ \\
\hline $\begin{array}{l}\text { Delay in administration } \\
\text { (interruption treatment) }\end{array}$ & $21(14.4)$ \\
\hline $\begin{array}{l}\text { Withdrawal treatment (oxaliplatine } \\
\text { withdrawn from regimen) }\end{array}$ & $21(14.4)$ \\
\hline Yes & $125(85.6)$ \\
\hline No & $21(14.4)$ \\
\hline
\end{tabular}


patients $(n=142,97 \%)$ had polychemotherapy based on combination of at least 2 cytotoxic drugs. Mostly, in the first line, chemotherapy regimen was based on XELOX or XELOX with bevacizumab protocol (in $73.3 \%$ of cases).

Chemotherapy-related toxicities and complications: Table 2 summarizes all toxicity events that occurred during chemotherapy for all the patients. The incidence rate of adverse events (grade I to IV) was $85.6 \%$. Acceptable toxicities were more observed than severe toxicities $(77.4 \%$ grade I-II, and $13 \%$ grade III-IV) (Table 3). Diarrhea was the predominant toxicity $(4.6 \%)$ occurring with a high grade (grade III-IV).

The most common grade I-IV (all grades) hematologic toxicities were neutropenia (33\%), anemia (3.2\%) and thrombopenia (1.6\%). The most common grade I-IV (all grades) non-hematologic toxicities were peripheral neuropathy (16\%), nausea/vomiting (48\%), diarrhea (45.6\%) and handfoot syndrome $(32 \%)$.
Due to toxicity occurrence, 21 patients (14.4\%) had dose delay, $14.4 \%$ had withdrawal of drug from the protocol (oxaliplatine withdrawn), 6.2\% required dose reduction during therapy, and $2.1 \%$ had premature discontinuation of chemotherapy.

\section{Comparison study of Chemotherapy} Toxicity and related factors: We studied the differences between the occurrence of toxic events and different demographic, clinicopathologic and therapeutic parameters. The statistic test did not reveal a significant difference, and the comparison with all parameters showed a $p>0.05$ (Table 3).

Univariate and multivariable logistic regression analyses were used, but revealed no significant differences in baseline demographic and clinical characteristics between the patients who encountered toxicities and the patients with no toxicity event notified.

Table 2: Chemotherapy-related toxicities reported in patients during treatment

\begin{tabular}{|c|c|c|c|}
\hline Toxicity type & $\begin{array}{l}\text { Grade I-IV } \\
\text { Number (\%) }\end{array}$ & $\begin{array}{c}\text { Grade I-II } \\
\text { Number (\%) }\end{array}$ & $\begin{array}{l}\text { Grade III-IV } \\
\text { Number ( \%) }\end{array}$ \\
\hline All types of toxicity & $125(85.6)$ & $113(77.4)$ & $19(13.0)$ \\
\hline Hematologic & & & \\
\hline $\begin{array}{l}\text { Neutropenia } \\
\text { Thrombopenia }\end{array}$ & $\begin{array}{c}45(36.0) \\
2(1.6)\end{array}$ & $\begin{array}{c}42(33.6) \\
2(1.6)\end{array}$ & $\begin{array}{l}4(3.2) \\
0(0.0)\end{array}$ \\
\hline Anemia & $4(3.2)$ & $2(1.6)$ & $2(1.6)$ \\
\hline $\begin{array}{l}\text { Gastrointestinal } \\
\text { Diarrhea } \\
\text { Nausea/vomiting }\end{array}$ & $\begin{array}{l}57(45.6) \\
60(48.0)\end{array}$ & $\begin{array}{l}51(40.8) \\
57(45.6)\end{array}$ & $\begin{array}{l}6(4.8) \\
4(3.2)\end{array}$ \\
\hline $\begin{array}{l}\text { Oropharyngeal } \\
\text { Mucositis }\end{array}$ & $30(24.0)$ & $29(23.2$ & $1(0.8)$ \\
\hline $\begin{array}{l}\text { Stomatitis } \\
\text { Gingivostomatitis }\end{array}$ & $\begin{array}{l}1(0.8) \\
1(0.8)\end{array}$ & $\begin{array}{l}1(0.8) \\
1(0.8)\end{array}$ & $\begin{array}{l}0(0.0) \\
0(0.0)\end{array}$ \\
\hline Dermatological & & & \\
\hline Dermatitis & $1(0.8)$ & $(0.8)$ & $0(0.0)$ \\
\hline Alopecia & $3(2.4)$ & $3(2.4)$ & $0(0.0)$ \\
\hline Hand and foot syndrome & $40(32.0)$ & $40(32.0)$ & $0(0.0)$ \\
\hline $\begin{array}{l}\text { Neurologic } \\
\text { peripheral neuropathy }\end{array}$ & $76(60.8)$ & $72(57.6)$ & $4(3.2)$ \\
\hline
\end{tabular}

DOI: http://dx.doi.org/10.4314/ejhs.v30i1.9 
Therapeutic Characteristics, Chemotherapy...

Zineb A. et al.

Table 3: Relationship between patients' characteristics and toxicity occurrence

\begin{tabular}{|c|c|c|c|}
\hline Variables & $\begin{array}{c}\text { No toxicity } \\
\mathrm{N}(\%)\end{array}$ & $\begin{array}{c}\text { Toxicity } \\
\text { N (\%) }\end{array}$ & $p$ \\
\hline \multicolumn{4}{|l|}{ Localisation } \\
\hline Colon & $9(42.9)$ & $60(48.0)$ & \multirow[t]{2}{*}{0.66} \\
\hline Rectum & $12(57.1)$ & $65(52.0)$ & \\
\hline \multicolumn{4}{|l|}{ Surgery } \\
\hline No & $7(33.3)$ & $31(24.8)$ & \multirow[t]{2}{*}{0.41} \\
\hline Yes & $14(66.7)$ & $94(75.2)$ & \\
\hline \multicolumn{4}{|l|}{ CCR } \\
\hline No & $16(76.2)$ & $96(76.8)$ & \multirow[t]{2}{*}{1} \\
\hline Yes & $5(23.8)$ & $29(23.2)$ & \\
\hline \multicolumn{4}{|l|}{ RTH } \\
\hline No & $16(76.2)$ & $96(76.8)$ & \multirow[t]{3}{*}{0.53} \\
\hline neoadjuvant & $4(19.0)$ & $27(21.6)$ & \\
\hline exclusive & $1(4.8)$ & $2(1.6)$ & \\
\hline \multicolumn{4}{|l|}{ CMT } \\
\hline adjuvant & $8(38.1)$ & $42(33.6)$ & \multirow[t]{4}{*}{0.74} \\
\hline neoadjuvant & $0(0)$ & $6(4.8)$ & \\
\hline pseudoadjuvant & $0(0)$ & $8(6.4)$ & \\
\hline palliative & $13(61.9)$ & $69(56.2)$ & \\
\hline \multicolumn{4}{|l|}{ CMT lines } \\
\hline 1 line & $18(81.8)$ & $83(69.7)$ & \multirow{3}{*}{0.12} \\
\hline 2 lines & $3(13.6)$ & $33(27.7)$ & \\
\hline 3 lines & $1(4.5)$ & $3(2.5)$ & \\
\hline \multicolumn{4}{|c|}{ Type of Chemotherapy } \\
\hline Mono & $1(4.8)$ & $3(2.4)$ & \multirow[t]{2}{*}{0.46} \\
\hline Poly & $20(95.2)$ & $122(97.6)$ & \\
\hline
\end{tabular}

Survival and outcome: Figure 1 depict the results of Kaplan-Meier analysis. The 3-year overall survival rate of patients with colorectal cancer was $71 \%$. Median follow-up was 14 months (14-34) with a range of $0-108$ months.

In our series, overall 3-year survival decreased by age. Patients aged 50 years or older exhibited a higher mortality rate than patients aged less than 50 years with a p-value of 0.04 . The patients belonging to the age subgroup of between
40 to 59 years had a better overall survival than the other age subgroups ( 60 to 79 years and $>80$ years) with a p-value of 0.0001 (Figure 1a). Survival rate was lower for patients with distant metastasis at diagnosis (p-value of 0.0001), and patients diagnosed with at least one metastatic site than patients with local tumor (p-value of 0.0001) (Figure $1 \mathrm{~b} \& 1 \mathrm{c}$ ). Patients with stage IV at diagnosis have a reduced OS, compared to stage II and III. (Fig 1d). 
The study of the relationship between treatment and the overall survival reveals that patients who had a polychemotherapy had a better OS than those who had monotherapy (p-value of 0.002). For patients with monotherapy, the survival was high up to 22 months, then dropped to about $65 \%$ (Figure 1e). Moreover, a pseudoadjuvant chemotherapy gave a better OS than the other chemotherapeutic types ( $p$-value of 0.002 ) (Figures 1e \& 1f). We also noted a better survival rate for patients with 2 lines of chemotherapy than one-line chemotherapy or none (p-value of 0.002).

The occurrence of toxicity (all grades and types) was linked to a higher survival compared to the group who had no toxicity noticed (p-value of 0.001) (Figure 1g). For rectum cancer patients, the
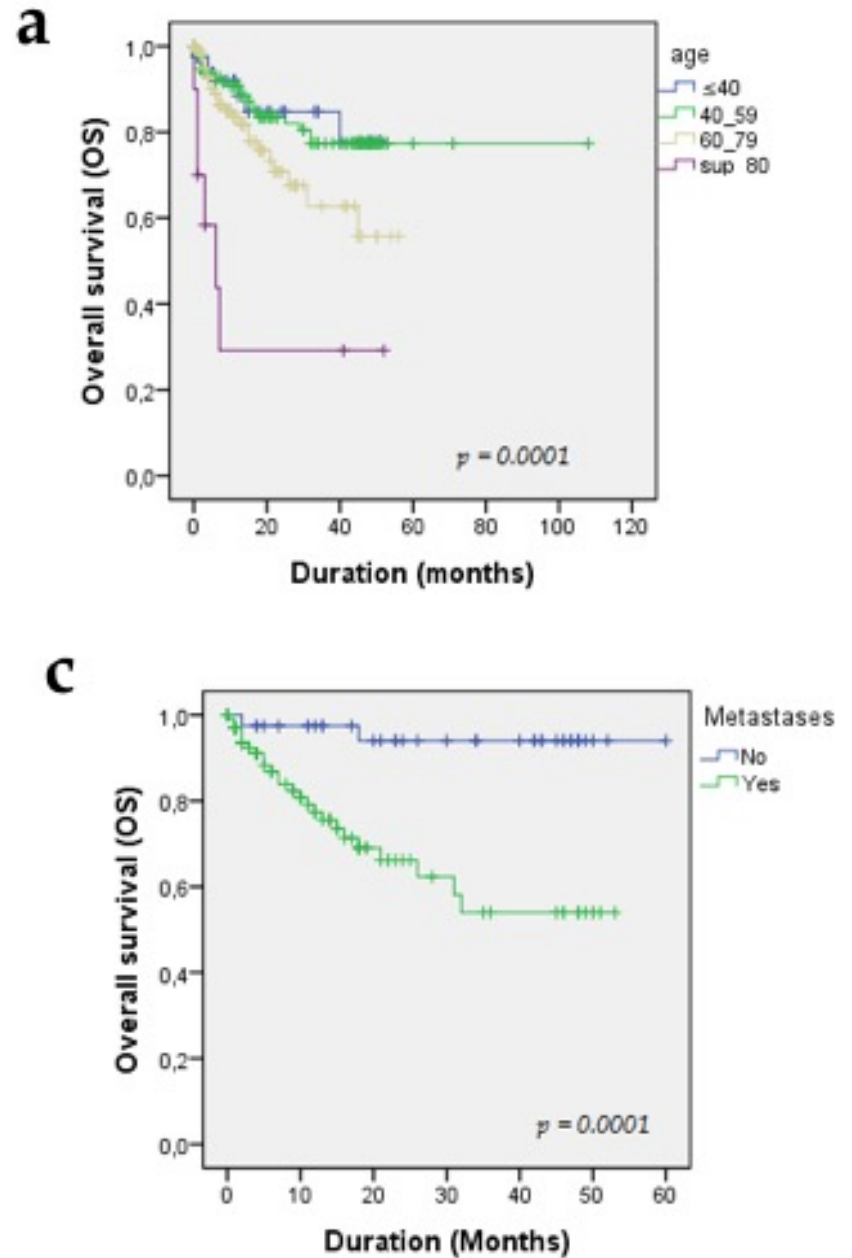

Figure 1: Overall survival in colorectal cancer patients
OS was significantly higher when chemoradiation therapy was administered ( $\mathrm{p}$-value of 0.012) (Figures $1 \mathrm{~g} \& \mathrm{~h}$ ), and with a neoadjuvant radiotherapy, the OS increased significantly than an exclusive radiotherapy (p-value of 0.02) (Figure 1i).

A significant difference in OS was noted between patients who underwent a surgery and non-operated patients with a better overall survival for the first group.

There was no significant difference in KaplanMeier analysis, in overall survival between colon localization and rectum $(p=0.07)$ and between different tumor localizations in colon ( $\mathrm{p}$ value of 0.07).
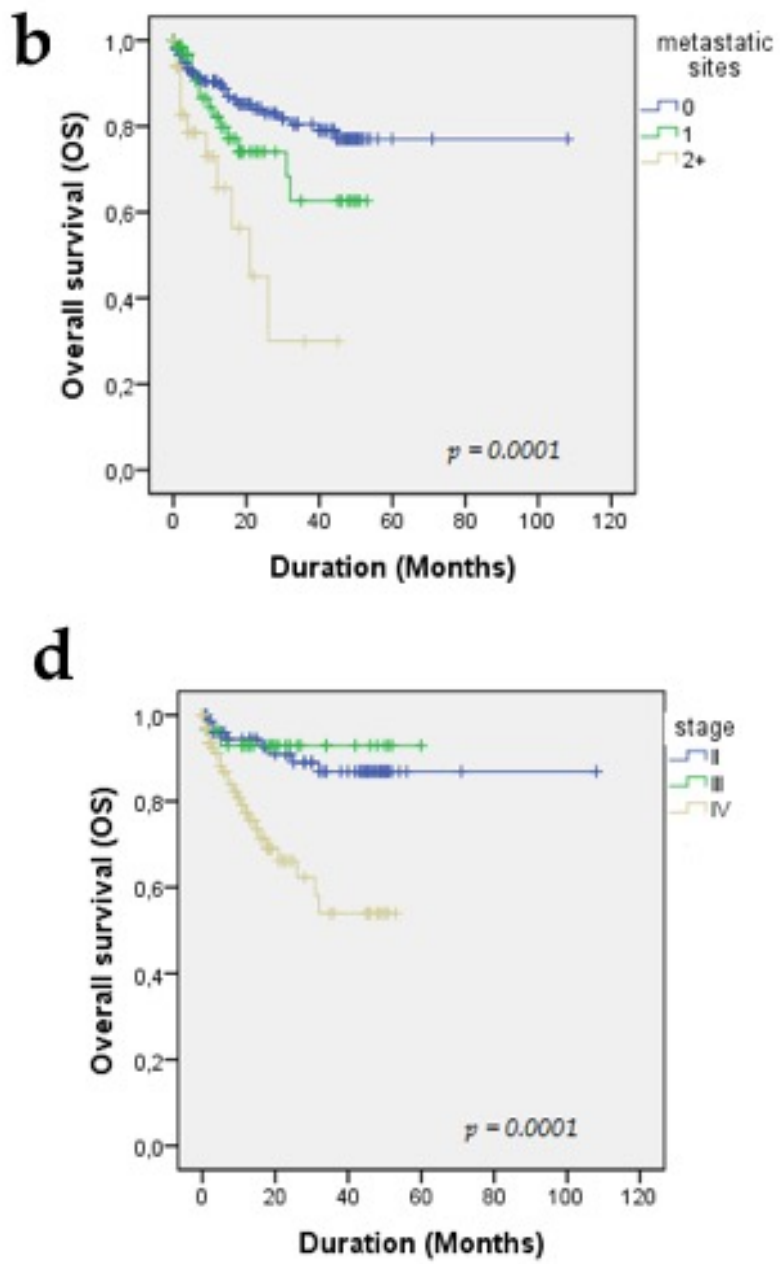
e

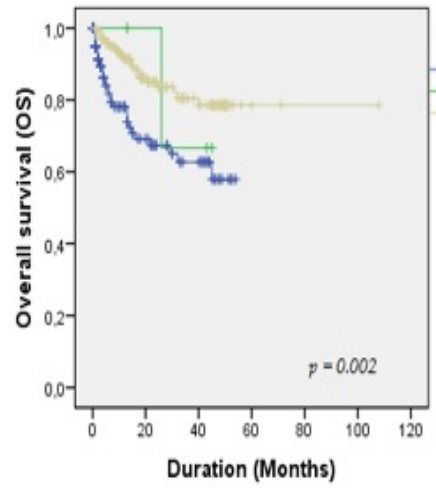

h

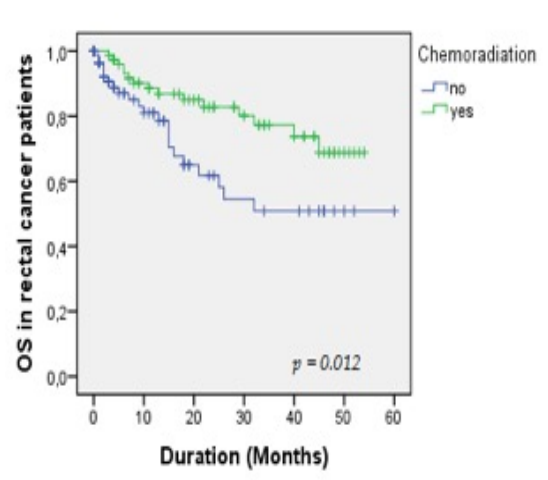

f

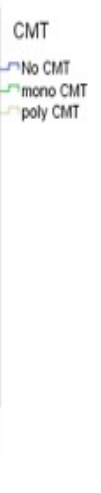

h

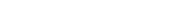

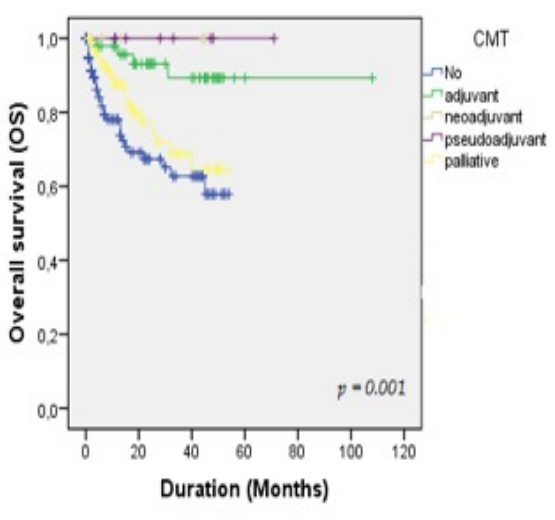

i

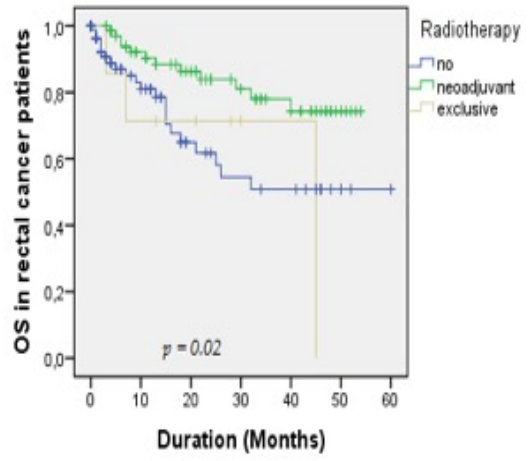

g

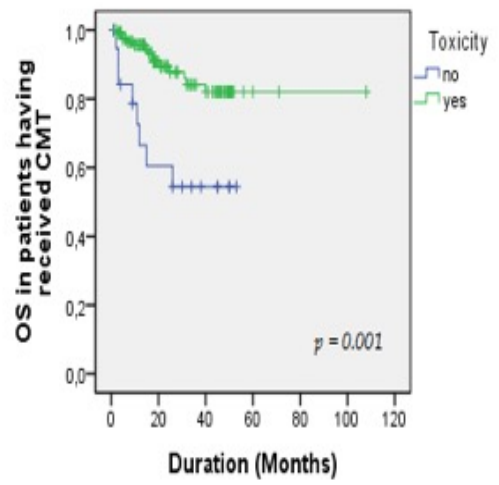

j

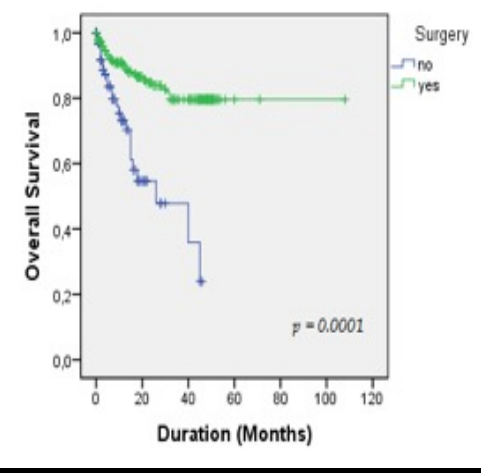

Figure 1: Overall survival in colorectal cancer patients (continued)

$K e y=a$-relationship between age (age ranges) and the overall survival (OS), $b$ - relationship between tumor extension (metastatic sites) and the OS, $c$-relationship between presence of metastasis and the OS, $d$-relationship between stage of the tumor at diagnosis and the OS, $e$-relationship between treatment by chemotherapy and OS, $f$-relationship between types of chemotherapy and the OS, $g$ - relationship between occurrence of toxicity and the OS, $h$ - relationship between treatment by chemoradiation and the OS, $i$ - relationship between treatment by radiotherapy and the OS, $j$ - relationship between treatment by surgery and the OS.

\section{DISCUSSION}

We were interested to investigate clinical, pathological and therapeutic characteristics and outcomes. Overall survival was also an interesting feature to analyze.

In our population study, only $15.2 \%$ were aged less than 40 years for both males and females; a similar rate was found by a previous study (5). As previously described, before the age of 40 years, developing colorectal cancer is rare (6), and the risk is sharply increasing with age (7).

On patients' sex, the distribution of colorectal cancer shows a balanced sex ratio, with a slight male predominance $(50.5 \%$ males vs $49.5 \%$ females). This is in agreement with previous studies $(5,8-10)$.

In our series, $57 \%$ of those cancers interested the rectum and the colon in $42.8 \%$ of cases. This revealed that predilection of the rectum is in agreement with previous Moroccan epidemiological studies (5), the Moroccan registries and African 
cohort studies, but in discordance with the rate reported by other European and American studies which noted a predominance of colic tumor localization $(11,12)$.

The diagnosis of CRC was in advanced stage for $48.6 \%$ of cases, otherwise $51.4 \%$ of tumors are discovered at a relatively early stage $(37 \%$ in stage II, and $14.5 \%$ in stage III). However, the cancer registry of Rabat (3) reported that "Colon cancer is often diagnosed in advanced stages: a quarter of cases in stage III and one third of cases in stage IV" and for rectal localization "Rectal: more than a quarter of cases are diagnosed at stage IV", contradictory to developed countries where the earlier stages, I and II, are the most frequent (13).

To our knowledge, this is the first retrospective study reporting toxic events related to chemotherapy in Morocco. A detailed description of grades and toxicity types and this one of the several strengths of our study. Reporting toxic events is a key component of oncological cohort studies and randomized controlled trials (RCTs) to evaluate patient safety, to improve clinicians' understanding of toxicity and to assess risk-benefit ratios (14). In addition, even for international investigations, limited data are available on the burden represented by all (including low-grade) adverse events (AEs) experienced during systemic treatment with chemotherapy (15-17).

In our population study, only 146 underwent treatment by chemotherapy, among these, a total of $125(85.6 \%)$ patients suffered from at least one toxicity (any grade), 113(77.4\%) patients experienced exclusively low-grade (I-II) toxicities, and $19(13 \%)$ patients experienced at least one highgrade toxicity (III-IV). The most common toxicities (all grades) were neuropathy $(60.8 \%)$, nausea and vomiting (48\%) and diarrhea (45.6\%). These frequencies are comparable to those reported recently by Schuurhuizen et al., 2018 (18).

A previous prospective cohort study of older adults with cancer reported that $53 \%$ of patients suffered from at least one grade III to V toxicity (39\% grade III, $12 \%$ grade IV, and $2 \%$ grade V (chemotherapy-related death). The most common grade III to $\mathrm{V}$ hematologic toxicities were neutropenia (11\%). $31 \%$ of patients required a dose reduction during therapy, $31 \%$ had a dose delay, and $23 \%$ were hospitalized during treatment (19).
According to NCI-CTCAE, physicians grade toxicities and all adverse events (AEs) from low to severe (I to V). Grades III and IV commonly urge for clinical action(18).

Indeed, lower grade toxic effects are considered as tolerable and often neglected by the clinical trials and RCTs limiting reporting to severe toxicities only for the total study population (20). Nevertheless, the Quality of Life (QOL) of the patient is impacted by these long-lasting toxicities even with low grade. Furthermore, a recent cohort study by KalsiT et al., 2014 (21) reported that the accumulation of solely grades I-II AEs, considered as low-grade toxicities, have clinically impacted older patients, leading to discontinuation of their treatment or at least a treatment modification (21).

In our series, we reported the impact of toxicity occurrence (for high grades) on patients' treatment; $2.1 \%$ had firmly premature chemotherapy discontinuation, $14.4 \%$ had withdrawal of drug from the protocol, $14.4 \%$ had dose delay while $6.2 \%$ required a drug dose reduction.

In our series, the 3-year overall survival rate of patients with colorectal cancer was $71 \%$. In a previous study by ASEVOAIA, 2010 (22), the 5year survival rate was $10 \%$. In France, the 5-year overall survival rate is about $55 \%(23)$.

In colorectal cancer, locally advanced or metastatic, chemotherapy increases survival and the patient's quality of life. It was reported that the 5year survival rate increased by $3 \%-4 \%$ when 5 fluorouracil (5-FU)-based adjuvant chemotherapy was administered to patients with stage II colon cancer and by $5 \%-12 \%$ for patients with stage III colon cancer when compared with surgery alone (24).

The combination of several molecules in a chemotherapeutic protocol represents the mainstay of the treatment of metastatic colorectal cancer. It may be responsible for an increase in response rate, as well as an increase in patient survival. Interaction analysis showed that the magnitude of risk reduction in death from CRC $(\mathrm{P}<0.0001)$ significantly differed between chemotherapy recipients and nonrecipients (25).

Current combination therapies have been favored over monotherapy strategies as a result of improved response rates, Overall Survival (OS) and Progression-Free Survival (PFS) rates. This is in

DOI: http://dx.doi.org/10.4314/ejhs.v30i1.9 
accordance with our study findings as we found that the overall survival reveals that patients who had polychemotherapy had better OS than those who had monotherapy (p-value of 0.002).

In addition, we found that for rectum cancer patients, neoadjuvant chemoradiotherapy increased significantly the OS than an exclusive chemoradiotherapy ( $p$-value of 0.02 ) which is in agreement with a controlled study on the survival of patients with rectal cancers (26). Neoadjuvant irradiation reduces the risk of loco-regional recurrence and increased patient survival.

Tong et al. found that patients experiencing hematological toxicity were less likely to die of CRC $(0.79,0.70-0.88)$ than those without, and that patients who experienced gastric toxicity were less likely to die of CRC $(0.80,0.77-0.84)$ than those without (19), similar to the finding of our study where we report that the occurrence of toxicity (all grades and types) was linked to a higher survival compared to the group who had no toxicity noticed (p-value of 0.001).

To date, this is the first investigation assessing chemotherapy-related toxicities among patients under chemotherapy regimens. Because of the lack of data on this topic, our study adds relevant information and gives reliable figures about the side effects of chemotherapy in CRC patients. It shows that $85,6 \%$ of the patients suffered from at least one toxicity (any grade), and 13\% of the patients experienced at least one high-grade toxicity (III-IV), which could not be neglected and should be considered as a good indicator of chemotherapy tolerability among this population of Moroccan colorectal patients.

The analysis of the OS is another strength of the study giving a clear idea about CRC patients' life expectancy in relation with several conditions such as stage of the disease, chemotherapy regimen combinations and type of treatment underwent. Thus, we found that the 3 -year overall survival rate of patients with colorectal cancer was $71 \%$ and that the OS was influenced by chemotherapy administered, in other words, patients who had a polychemotherapy had a better OS than those who had monotherapy (p-value of 0.002).

\section{ACKNOWLEDGEMENTS}

We thank the team from the epidemiology unit at the National Institute of Oncology for providing us with necessary medical records needed for the study.

\section{REFERENCES}

1. Bray F, Ferlay J, Soerjomataram I. Global Cancer Statistics 2018: GLOBOCAN Estimates of Incidence and Mortality Worldwide for 36 Cancers in 185 Countries. CA CANCER J CLIN. 2018;00(00):1-31.

2. Casablanca G. REGISTRE DES CANCERS. 2016.

3. Registre Cancer Rabat. 2012.

4. Pirmohamed M. Personalized Pharmacogenomics: Predicting Efficacy and Adverse Drug Reactions. Annu Rev Genomics Hum Genet. 2014;15(1):349-70.

5. Imane Allali et al. A Moroccan Colorectal Cancer Database Imane. Int J Bio-Medical Informatics e-Health. 2017;5(3):10-4.

6. Chiang JM, Chen MC, Changchien CR, Chen JS, Tang R, Wang JY, et al. Favorable influence of age on tumor characteristics of sporadic colorectal adenocarcinoma: Patients 30 years of age or younger may be a distinct patient group. Dis Colon Rectum. 2003;46(7):904-10.

7. Pinsky PF, Schoen RE. Colorectal cancer incidence by age among patients undergoing surveillance colonoscopy. JAMA Intern Med. 2015;175(5):858-60.

8. Hannan LM, Jacobs EJ, Thun MJ. The association between cigarette smoking and risk of colorectal cancer in a large prospective cohort from the United States. Cancer Epidemiol Biomarkers Prev. 2009;18(12):3362-7.

9. Teresa T. Fung \& Lisa S. Brown. Dietary Patterns and the Risk of Colorectal Cancer. Curr Nutr Rep. 2013 Nov;2:48-55.

10. Weikert C, Dietrich T, Boeing H, Bergmann MM, Boutron-Ruault MC, Clavel-Chapelon $\mathrm{F}$, et al. Lifetime and baseline alcohol intake and risk of cancer of the upper aero-digestive tract in the European prospective

DOI: http://dx.doi.org/10.4314/ejhs.v30i1.9 
investigation into cancer and nutrition (EPIC) study. Int $J$ Cancer. 2009;125(2):406-12.

11. Purim O, Gordon N, Brenner B. Cancer of the colon and rectum: Potential effects of sex-age interactions on incidence and outcome. Med Sci Monit. 2013;19(1):203-9.

12. Stigliano V, Sanchez-Mete L, Martayan A, Anti M. Early-onset colorectal cancer: A sporadic or inherited disease? World $J$ Gastroenterol. 2014;20(35):12420-30.

13. Arndt V, Merx H, Stegmaier C, Ziegler H, Brenner H. Quality of life in patients with colorectal cancer 1 year after diagnosis compared with the general population: A population-based study. $J$ Clin Oncol. 2004;22(23):4777-84.

14. Seruga B, Templeton AJ, Badillo FEV, Ocana A, Amir E, Tannock IF. Underreporting of harm in clinical trials. The Lancet Oncology. 2016;17: e209-19.

15. Koehler M, Fischer T, Kropf S, Frommer J. Quantitative tool to evaluate the somatic burden due to chemotherapy-induced adverse events: the somatic burden score. Support Care Cancer. 2014;22(11):308999.

16. Péron J, Maillet D, Gan HK, Chen EX, You B. Adherence to CONSORT adverse event reporting guidelines in randomized clinical trials evaluating systemic cancer therapy: A systematic review. Journal of Clinical Oncology. 2013;31:3957-63.

17. Pitrou I, Boutron I, Ahmad N, Ravaud P. Reporting of safety results in published reports of randomized controlled trials. Arch Intern Med. 2009;169(19):1756-61.

18. Schuurhuizen CSEW, Verheul HMW, Braamse AMJ, Buffart LM, Bloemendal HJ, Dekker J, et al. The predictive value of cumulative toxicity for quality of life in patients with metastatic colorectal cancer during first-line palliative chemotherapy. Cancer Manag Res. 2018;10:3015-21.

19. Hurria A, Togawa K, Mohile SG, Owusu C,
Klepin HD, Gross CP, et al. Predicting chemotherapy toxicity in older adults with cancer: A prospective multicenter study. $J$ Clin Oncol. 2011;29(25):3457-65.

20. Gilbert A, Ziegler L, Martland M, Davidson $\mathrm{S}$, Efficace F, Sebag-Montefiore D, et al. Systematic review of radiation therapy toxicity reporting in randomized controlled trials of rectal cancer: A comparison of patient-reported outcomes and clinician toxicity reporting. International Journal of Radiation Oncology Biology Physics. 2015;92:555-67.

21. Kalsi T, Babic-Illman G, Fields P, Hughes S, Maisey N, Ross P, et al. The impact of lowgrade toxicity in older people with cancer undergoing chemotherapy. $\mathrm{Br} J$ Cancer. 2014;111(12):2224-8.

22. Doctorat $\mathrm{T}$ De. Caractérisation pharmacogénétique des facteurs de résistance et de toxicité de 1' Oxaliplatine et de 1' Irinotécan dans les tumeurs colorectales. 2010;

23. Seitz JF, Houvenaeghel G, Dahan L. Tumeurs du colon et du rectum (148). In 2007.

24. Gray RG, Kerr DJ, McConkey CC, Williams NS, Hills RK. Comparison of fluorouracil with additional levamisole, higher-dose folinic acid, or both, as adjuvant chemotherapy for colorectal cancer: A randomised trial. Lancet. 2000;355(9215):1588-96.

25. Tong L, Ahn C, Symanski E, Lai D, Du XL. Effects of newly developed chemotherapy regimens, comorbidities, chemotherapyrelated toxicities on the changing patterns of the leading causes of death in elderly patients with colorectal cancer. Ann Oncol. 2014;25(6):1234-42.

26. Cedermark B, Johansson H, Rutqvist LE, Nils Wilking N. The Stockholm I trial of preoperative short term radiotherapy in operable rectal carcinoma. Cancer. 1995;75(9):2269-75. 